\title{
Physiological values of ECG parameters in Silesian horses
}

\author{
URSZULA PASŁAWSKA, KATARZYNA MICHLIK*, IZABELA JANUS**, \\ ROBERT PASŁAWSKI***, DOROTA ZYŚKO****, AGNIESZKA NOSZCZYK-NOWAK
}

\author{
Department of Internal Diseases with Clinic for Horses, Dogs, and Cats, Faculty of Veterinary Medicine, \\ Wrocław University of Environmental and Life Sciences, pl. Grunwaldzki 47, 50-366 Wrocław, Poland \\ *Department of Internal Diseases and Veterinary Diagnostics, \\ Poznań University of Life Sciences, Wołyńska 35, 60-637 Poznań, Poland \\ **Department of Pathology, Faculty of Veterinary Medicine, \\ Wrocław University of Environmental and Life Sciences, Norwida 31, 50-375 Wrocław, Poland \\ ***Department and Clinic of Internal and Occupational Diseases and Hypertension, and Clinical Oncology, \\ Wrocław Medical University, Borowska 213, 50-556 Wrocław, Poland \\ ****Department of Emergency Medicine, Wrocław Medical University, Borowska 213, 50-556 Wrocław, Poland
}

\section{Pasławska U., Michlik K., Janus I., Pasławski R., Zyśko D., Noszczyk-Nowak A.}

\section{Physiological values of ECG parameters in Silesian horses}

\section{Summary}

The aim of this study was to establish the physiological ECG values in the Silesian horse and to compare the results with literature data for other breeds of horses. This study was carried out on 31 healthy Silesian horses (15 males and 16 females) aged 2-19 years. The ECG was carried out after an anamnesis and clinical examination and without prior premedication or use of force, both of which could affect the ECG result. The mean heart rate (HR) in the Silesian horses was $39 \pm 8.1$ beats per minute. In the majority of the leads, $P$ waves were single positive waves. Notched waves were present in $16-51 \%$ of the horses, bifid waves were found less frequently, and biphasic $P$ waves were seen least frequently. In the aVR lead, the $P$ wave was most commonly found to be single negative. The PQ interval ranged from $0.18 \mathrm{~s}$ in the CV1 lead to $0.29 \mathrm{~s}$ in the aVR lead. Most often, the PQ interval lasted 0.24-0.28 s. The QRS duration ranged from $0.079 \mathrm{~s}$ in the CV1 lead to $0.099 \mathrm{~s}$ in lead III. The QRS duration was between 0.08 and $0.1 \mathrm{~s}$. Generally, the T wave was single positive or negative. It was biphasic only in the precordial leads. Unlike $P$ waves, notched $T$ waves were not found. The ECG parameters in the Silesian horse do not differ from normal ECG values for other horse breeds of similar weight. Specific features of the ECG in the Silesian horse are a frequent occurrence of additional R' and S' waves in the Einthoven leads and a relatively long supraventricular conduction time.

Keywords: ECG, horse, Silesian horses

The Silesian horse is a native Polish breed established in the late nineteenth century. It was initiated by using Oldenburg and Ostfriesen sires bred to a local population of mares. In the $1930 \mathrm{~s}$, there were 3000000 Silesian horses, $91 \%$ of which were used in agriculture. Their head profile and body mass were similar to those of the Oldenburg horse - they had a barrel-chested torso, a short and straight back, a horizontal rump and a high neck. They also were less picky eaters than the Oldenburg breed. After World War II, Silesian horses were crossbred with Thoroughbreds and Hanoverians, which permitted them to be used in sports.

To date, no electrocardiographic studies determining the normal ECG ranges of the Silesian horses have been performed. Such norms have been determined for the Polish Konik (13) and Angloarab horses in Poland (14).

The aim of this study was to establish the physiological ECG value in the Silesian horse and to complement the knowledge of normal ECG features of Polish horses. Thus far, Polish Konik (13) and Anglo-Arab (14) breeds have been described, but Silesian horses have a greater body weight, which as we know, could affect the ECG parameters. Additionally, the ECG results will be compared with literature data for other breeds of horses.

\section{Material and methods}

This study was carried out on 31 healthy Silesian horses (15 males and 16 females) aged 2-19 years. The ECG was 
carried out after an anamnesis and clinical examination and without prior premedication or use of force, both of which could affect the ECG result.

The ECG was carried out in the morning between 9:00 and 11:00 am after a night's rest, according to the formerly described methodology $(13,14)$. A 3-channel Schiller AT-1 ECG was used. Leads I, II, III, aVR, aVL, aVF and precordial leads (CV1,
Tab. 1. The incidence of individual $P$ wave shapes $(\%)$

\begin{tabular}{|l|c|c|c|c|c|c|c|c|c|}
\hline P wave & I & II & III & aVR & aVL & aVF & CV1 & CV2 & CV3 \\
\hline Single & 44.80 & 45.20 & 65.40 & 45.20 & 56.50 & 55.20 & 66.70 & 64 & 65.40 \\
Bifid & 17.20 & 3.20 & 19.20 & 0 & 26.10 & 10.30 & 13.30 & 12 & 7.70 \\
Notched & 38 & 48.40 & 15.40 & 51.60 & 17.40 & 31 & 20 & 16 & 26.90 \\
Biphasic & 0 & 3.20 & 0 & 3.20 & 0 & 3.50 & 0 & 8 & 0 \\
\hline
\end{tabular}

Tab. 2. The incidence of individual $T$ wave shapes (\%)

\begin{tabular}{|l|c|c|c|c|c|c|c|c|c|}
\hline T wave & I & II & III & aVR & aVL & aVF & CV1 & CV2 & CV3 \\
\hline Single & 58.10 & 46.40 & 83.90 & 63.30 & 83.90 & 83.90 & 82.80 & 41.90 & 35.50 \\
Bifid & 6.40 & 0 & 0 & 0 & 0 & 3.20 & 0 & 0 & 0 \\
Notched & 0 & 3.60 & 3.20 & 0 & 0 & 0 & 3.40 & 0 & 0 \\
Biphasic & 35.50 & 50 & 12.90 & 36.70 & 16.10 & 12.90 & 13.80 & 58.10 & 64.50 \\
\hline
\end{tabular}

Crocodile clip electrodes were attached to skin folds (previously moistened and cleansed with alcohol) at the elbows, knee joints, on the chest, on both sides of the sternum in the $5^{\text {th }}$ intercostal space above the elbow and on the sternum at the level of the $5^{\text {th }}$ intercostal space. The mean heart rate in the group was calculated. The duration and amplitude of P, Q, R, S and T waves, the QRS complex as well as the PQ and QT interval duration were calculated for each lead, and the normal sinus rhythm was determined. The following shapes of the $\mathrm{P}$ and $\mathrm{T}$ waves were recorded: single, notched, bifid and biphasic.

The data is presented in tables as arithmetic means with standard deviations. Modal values were calculated for individual waves and intervals.

\section{Results and discussion}

Horses were initially divided into two age-related groups (less than 10 years old and at least 10 years old), but there were no significant differences in ECG values, so all horses were considered as a single group.

The mean heart rate in the Silesian horses was $39 \pm 8.1 \mathrm{bpm}$, with the modal value amounting to $40 \mathrm{bpm}$, which falls within the normal limits for adult horses (15). The relatively low physiological HR in horses is a result of high parasympathetic tone in this species (the highest of all animals), which inhibits the intrinsic heart rate that ranges from 74 to $110 \mathrm{bpm}$. This has been documented in an experimental study, in which functional denervation of the equine heart was induced by a simultaneous application of a betaadrenergic and a cholinergic blockade. The physiological vagal tone is higher in Thoroughbred horses than in ponies (9). A high parasympathetic resting tone causes supraventricular arrhythmias, such as the sinus arrhythmia, sino-atrial block, and first- and seconddegree atrioventricular (AV) block. Respiratory sinus arrhythmia was found in four of the examined horses. A second-degree Mobitz I-type AV block was found in an 8-year-old mare at rest, which subsided after a stress test (20 min of lunging). In the remaining 26 horses, a regular sinus rhythm was present. None of the horses had any pathological arrhythmias. The incidence of
Tab. 3. Modal values of wave durations and intervals (sec) measured in different leads

\begin{tabular}{|l|c|c|c|c|c|}
\hline \multicolumn{1}{|c|}{ Leads } & P & PQ & QRS & T & QT \\
\hline I & 0.10 & 0.28 & 0.10 & 0.12 & 0.50 \\
II & 0.12 & 0.28 & 0.1 & 0.13 & 0.52 \\
III & 0.08 & 0.28 & 0.09 & 0.12 & 0.49 \\
aVR & 0.12 & 0.28 & 0.08 & 0.16 & 0.48 \\
aVL & 0.08 & 0.28 & 0.08 & 0.12 & 0.48 \\
aVF & 0.12 & 0.28 & 0.08 & 0.12 & 0.48 \\
CV1 & 0.08 & 0.24 & 0.08 & 0.12 & 0.48 \\
CV2 & 0.08 & 0.24 & 0.10 & 0.16 & 0.48 \\
CV4 & 0.08 & 0.28 & 0.08 & 0.12 & 0.48 \\
\hline
\end{tabular}

arrhythmias in the study group of Silesian horses was lower than those reported in other breeds of horses. However, this may have been due to a short recording period (16). Significantly fewer arrhythmias can be detected during a short registration period, compared to a 24-hour Holter recording, because many arrhythmias have a paroxysmal character. Another reason for a relatively low incidence of resting arrhythmias was the fact that the most common heart frequency in those horses was 40 beats/min, whereas physiological arrhythmias occur at between 20-40 beats/min (11). Arrhythmias usually disappear above this frequency.

In the majority of the leads, the $P$ waves were single positive waves. Notched waves were present in $15-52 \%$ of the horses (depending on the lead, tab. 1), bifid waves were found less frequently, and biphasic $\mathrm{P}$ waves were seen least frequently. In the aVR lead, the $\mathrm{P}$ wave was most commonly found to be single negative.

The P-wave conformation described by different authors varies depending on the lead system used, heart rate and age. Generally, a bifid $\mathrm{P}$ wave is more frequent than a simple $\mathrm{P}$ wave in animals older than 6 months. This phenomenon occurs in combination with a decrease in heart rate observed during this period (1). A simple positive conformation is associated with an activation of the sympathetic nervous system. 
Therefore, in practice, it is most commonly observed in horses after exercise or in other situations associated with increased HR (12). In our study, the P wave was mainly simple positive, notched or bifid. The relatively small number of biphasic $\mathrm{P}$ waves and the relatively high resting HR in the adult Silesian horses in the present study may be explained by the animals' excitement during the measurement despite the authors' efforts to perform the ECG in stress-free conditions.

Alaya et al. $(1,2)$ reported a correlation between the $\mathrm{P}$ wave amplitude and age. Waves with the highest amplitude occurred in young horses between 6 months and 2 years of age. Such a tendency was not observed in the Silesian horses, although horses under 2 years of age were not examined in this study.

In our study the PQ interval ranged from $0.18 \mathrm{~s}$ in the $\mathrm{CV}$ lead to $0.29 \mathrm{~s}$ in the aVR lead (Tab. 4). Most often, the PQ interval duration was $0.24-0.28 \mathrm{~s}$ (Tab. 3). The QRS duration ranged from $0.079 \mathrm{~s}$ in the CV lead to $0.099 \mathrm{~s}$ in lead III (Tab. 3). Generally, the T wave was single positive or negative. It was biphasic only in the precordial leads. In contrast to the $\mathrm{P}$ waves, notched $\mathrm{T}$ waves were detected only in leads II, III and CV1 (Tab. 2).

PQ intervals were longer in the Silesian horse than in other breeds (Polish Konik, Andalusian horse) $(12,13)$, while the QRS and QT intervals were of a similar duration, which shows a longer atrial or atrioventricular node conduction time.

The QRS segment duration depends on the amount of muscular tissue and extends with an increasing mass of the heart muscle - secondary to body mass and in physiologic and pathologic hypertrophy (6). This relationship is visible in various breeds of horses in Poland. In the Silesian horses measuring
$165-175 \mathrm{~cm}$ at the withers, the QRS interval in lead II was $0.093 \mathrm{~s}$, that is, significantly longer than in the Polish Konik horses (QRS interval $=0.067 \mathrm{~s})$, measuring $130-140 \mathrm{~cm}$ at the withers (13).

Ventricular R' waves (in leads II, aVR, aVL, aVF and CV1) and S' waves (aVR, aVL) were frequently noted.

The polarity of the $T$ wave essentially depends on the leads in which the ECG was recorded, but changes markedly with growth (8), human contact (7), twitch restraint (10), exercise, training, fear and coronary flow (1). In general, an increase in the amplitude and a positive $T$ wave were associated with sympathetic stimulation. This phenomenon was experimentally confirmed by Matsui and Sagano (9). T-wave amplitude alters with functional variations in the autonomic tone,

Tab. 4. Modal values of wave amplitudes $(\mathrm{mV})$ measured in different leads

\begin{tabular}{|c|c|c|c|c|c|c|c|c|c|}
\hline Leads & $\mathrm{Pa}+$ & $\mathrm{Pa}-$ & $a$ & $\mathbf{R}$ & $\mathbf{R}^{\prime}$ & $S$ & $\mathbf{S}^{\prime}$ & $\mathrm{Ta}+$ & Ta- \\
\hline I & 0.15 & 0 & 0 & 0.4 & 0 & 0.1 & 0 & 0 & 0.3 \\
\hline II & 0.2 & 0 & 0 & 0.7 & 0 & 0.1 & 0 & 0.2 & 0.1 \\
\hline III & 0.1 & 0 & 0 & 0.2 & 0 & 0 & 0 & 0.4 & 0 \\
\hline aVR & 0 & 0.2 & 0.2 & 0.1 & 0 & 0 & 0 & 0.2 & 0.1 \\
\hline aVL & 0 & 0 & 0 & 0.2 & 0 & 0 & 0 & 0 & 0.3 \\
\hline aVF & 0.15 & 0 & 0 & 0.55 & 0 & 0 & 0 & 0.3 & 0 \\
\hline CV1 & 0 & 0 & 0 & 0.2 & 0 & 0.15 & 0 & 0.1 & 0 \\
\hline CV2 & 0.1 & 0 & 0 & 0.15 & 0 & 0.4 & 0 & 0.2 & 0.2 \\
\hline CV4 & 0.15 & 0 & 0 & 0.2 & 0 & 0.25 & 0 & 0.15 & 0.1 \\
\hline
\end{tabular}

Tab. 5. Wave duration and intervals (sec) measured in different leads: mean values with standard deviations

\begin{tabular}{|l|c|c|c|c|c|}
\hline Leads & P & PQ & QRS & T & QT \\
\hline I & $0.09 \pm 0.036$ & $0.275 \pm 0.073$ & $0.096 \pm 0.027$ & $0.17 \pm 0.2$ & $0.075 \pm 0.04$ \\
II & $0.11 \pm 0.022$ & $0.286 \pm 0.043$ & $0.093 \pm 0.027$ & $0.16 \pm 0.06$ & $0.5 \pm 0.09$ \\
III & $0.082 \pm 0.026$ & $0.237 \pm 0.104$ & $0.1 \pm 0.029$ & $0.14 \pm 0.04$ & $0.49 \pm 0.05$ \\
aVR & $0.104 \pm 0.023$ & $0.29 \pm 0.05$ & $0.085 \pm 0.04$ & $0.16 \pm 0.07$ & $0.49 \pm 0.045$ \\
aVL & $0.086 \pm 0.03$ & $0.208 \pm 0.134$ & $0.083 \pm 0.024$ & $0.132 \pm 0.05$ & $0.47 \pm 0.046$ \\
aVF & $0.101 \pm 0.025$ & $0.26 \pm 0.084$ & $0.084 \pm 0.025$ & $0.13 \pm 0.045$ & $0.49 \pm 0.05$ \\
CV1 & $0.084 \pm 0.022$ & $0.18 \pm 0.136$ & $0.08 \pm 0.025$ & $0.13 \pm 0.13$ & $0.43 \pm 0.12$ \\
CV2 & $0.096 \pm 0.04$ & $0.247 \pm 0.095$ & $0.094 \pm 0.02$ & $0.14 \pm 0.054$ & $0.5 \pm 0.04$ \\
CV4 & $0.092 \pm 0.02$ & $0.243 \pm 0.108$ & $0.092 \pm 0.046$ & $0.135 \pm 0.04$ & $0.49 \pm 0.04$ \\
\hline
\end{tabular}

Tab. 6. Wave amplitude $(\mathrm{mV})$ measured in different leads: mean values with standard deviations

\begin{tabular}{|l|c|c|c|c|c|c|c|c|c|}
\hline Leads & P+ & P- & $Q$ & R & $R^{\prime}$ & $S$ & $S^{\prime}$ & $T_{+}$ & $T_{-}$ \\
\hline I & $0.19 \pm 0.01$ & $0 \pm 0$ & $0.06 \pm 0.1$ & $0.4 \pm 0.36$ & $0 \pm 0$ & $0.22 \pm 0.34$ & $0 \pm 0$ & $0.06 \pm 0.08$ & $0.37 \pm 0.24$ \\
II & $0.24 \pm 0.06$ & $0 \pm 0$ & $0.06 \pm 0.07$ & $0.724 \pm 0.35$ & $0.003 \pm 0.02$ & $0.12 \pm 0.15$ & $0 \pm 0$ & $0.25 \pm 0.15$ & $0.1 \pm 0.1$ \\
III & $0.12 \pm 0.11$ & $0.026 \pm 0.06$ & $0.05 \pm 0.08$ & $4.13 \pm 0.28$ & $0 \pm 0$ & $0.17 \pm 0.28$ & $0 \pm 0$ & $0.434 \pm 0.23$ & $0.02 \pm 0.05$ \\
aVR & $0.013 \pm 0.04$ & $0.2 \pm 0.08$ & $0.26 \pm 0.26$ & $0.14 \pm 0.12$ & $0.01 \pm 0.036$ & $0.215 \pm 0.37$ & $0.003 \pm 0.02$ & $0.214 \pm 0.2$ & $0.12 \pm 0.13$ \\
aVL & $0.08 \pm 0.09$ & $0.035 \pm 0.07$ & $0.18 \pm 0.32$ & $0.24 \pm 0.25$ & $0.014 \pm 0.05$ & $0.2 \pm 0.27$ & $0.013 \pm 0.04$ & $0.04 \pm 0.115$ & $0.38 \pm 0.34$ \\
aVF & $0.15 \pm 0.1$ & $0.02 \pm 0.06$ & $0.04 \pm 0.08$ & $0.61 \pm 0.35$ & $0.006 \pm 0.025$ & $0.1 \pm 0.18$ & $0 \pm 0$ & $0.3 \pm 0.17$ & $0.03 \pm 0.075$ \\
CV1 & $0.04 \pm 0.06$ & $0.01 \pm 0.03$ & $0.003 \pm 0.02$ & $0.22 \pm 0.24$ & $0.003 \pm 0.02$ & $0.2 \pm 0.18$ & $0 \pm 0$ & $0.16 \pm 0.115$ & $0.035 \pm 0.08$ \\
CV2 & $0.11 \pm 0.08$ & $0.01 \pm 0.04$ & $0.014 \pm 0.04$ & $0.22 \pm 0.25$ & $0 \pm 0$ & $0.46 \pm 0.36$ & $0 \pm 0$ & $0.15 \pm 0.1$ & $0.23 \pm 0.185$ \\
CV4 & $0.15 \pm 0.09$ & $0 \pm 0$ & $0.003 \pm 0.02$ & $0.24 \pm 0.2$ & $0 \pm 0$ & $0.38 \pm 0.39$ & $0 \pm 0$ & $0.18 \pm 0.1$ & $0.15 \pm 0.14$ \\
\hline
\end{tabular}


despite any clinical evidence of cardiac disease. On the other hand, in humans, T-wave orientation makes it possible to recognize myocardial disease or severe electrolyte imbalance. The analysis of the modal values obtained in all leads indicates that single positive, negative and biphasic waves were found equally frequently. This is a feature specific to horses.

The ECG parameters in the Silesian horse do not differ from normal ECG values for other horse breeds of similar weight. Specific features of the ECG in the Silesian horse are a frequent occurrence of additional R' and S' waves in leads I, II and III, and a relatively long supraventricular conduction time.

\section{References}

1. Ayala I., Gutierrez C., Benedito J. L., Hernández J., Castillo C., López A. M., Miranda M., Montes A.: Morphology and Amplitude Values of the P and T Waves in the Electrocardiograms of Spanish-Bred Horses of Different Ages. J. Vet. Med. 1999, 46, 225-230.

2. Ayala I., Gutierrez-Panizo C., Benedito J. L., Prieto F., Montes A.: Morphology and amplitude values of the electrocardiogram of Spanish-bred horses of different ages in the Dubois leads system. Vet. Res. 2000, 31, 347-354.

3. Brahmbhatt P. H., Jadhav K. M.: The electrocardiographic wave patterns in equine population (Equus caballus) of Gujarat state. Indian. J. Anim. Res. 2009, 43, 673-677.

4. Holmes J. R., Alps B. J.: Studies into equine electrocardiography and vectorcardiography II. Cardiac vector distributions in apparently healthy horses. Can. J. Comp. Med. Vet. Sci. 1967, 31, 150-155.
5. Holmes J. R., Rezakhani A.: Observation on the T-wave of the equine electrocardiogram. Equine Vet. J. 1975, 7, 55-62.

6. Lightowler C., Piccione G., Giudice E., del Olmo G. R., Cattaneo M. L. Echocardiography and electrocardiography as means to evaluate potential performance in horses. J. Vet. Sci. 2004, 5, 259-262.

7. Lynch J. J., Fregin G. F., Mackie J. B., Monroe R. R. Jr: Heart rate changes in the horse to human contact. Psychophysiology 1974, 11, 472-478.

8. Matsui K., Amada A., Sawasaki T., Kano Y.: Changes in electrocardiographic parameters with growth in thoroughbred horses and Shetland Ponies. Bull. Equine Res. Inst. 1983, 20, 77-86.

9. Matsui K., Sugano S.: Species differences in the changes in heart rate and T-wave amplitude after autonomic blockade in thoroughbred horsens, ponies, cows, pigs, goats and chickens. Jpn. J. Vet. Sci. 1987, 49, 637-644.

10. Matsui K., Sugano S., Amada A.: Heart rate and ECG response to twitching in thoroughbred foals and mares. Jpn. J. Vet. Sci. 1986, 48, 305-312.

11. Menzies-Gow N.: ECG interpretation in the horse. In Practice 2001, 9, 454 -459 .

12. Muñoz A., Castejón F., Rubio M. D., Tovar P., Santisteban R.: Electrocardiographic alterations in Andalusian horses associated with training. J. Equine Vet. Sci. 1995, 15, 72-79.

13. Pasławska U., Jaworski Z., Smolira M., Mrozowski T., Głazowska I., Jurusz M., Morawiecki A.: Obraz krzywej elektrokardiograficznej u koni rasy konik polski. Med. Weter. 2000, 56, 730-733.

14.Pasławska U., Pasławski R., Noszczyk-Nowak A., Michlik K., Nicpoń J.: Physiological ECG value for Polish half-bred Anglo-Arab horses. Bull. Vet. Inst. Pulawy 2012, 56, 631-635.

15. Patteson M.: Equine cardiology. Blackwell Science, Oxford 1996, p. 13.

16. Vibe-Petersen G., Nielsen K.: Electrocardiography in the horse. A report of findings in 138 horses. Nord. Vet. Med. 1980, 32, 105-121.

Corresponding author: DVM, PhD Katarzyna Michlik, Wolyńska 35, 60-637 Poznań, Poland; e-mail: katarzyna.michlik.wet@gmail.com 